\title{
Ricordo di Corrado Rosso
}

\section{(2) OpenEdition \\ 1 Journals}

\section{Edizione digitale}

URL: http://journals.openedition.org/studifrancesi/29808

DOI: 10.4000/studifrancesi.29808

ISSN: 2421-5856

\section{Editore}

Rosenberg \& Sellier

\section{Edizione cartacea}

Data di pubblicazione: 1 avril 2006

Paginazione: 5-6

ISSN: 0039-2944

\section{Notizia bibliografica digitale}

«Ricordo di Corrado Rosso», Studi Francesi [Online], 148 (XLX | I) | 2006, online dal 30 novembre 2015, consultato il 18 avril 2021. URL: http://journals.openedition.org/studifrancesi/29808 ; DOI: https:// doi.org/10.4000/studifrancesi.29808

\section{(c) (†) $\odot$}

Studi Francesi è distribuita con Licenza Creative Commons Attribuzione - Non commerciale - Non opere derivate 4.0 Internazionale. 


\section{Ricordo di Corrado Rosso}

Anche tu, Corrado, recentemente ci hai lasciati e anche a te mi rivolgo direttamente, come se fossi ancora tra noi. Sapevo della tua malattia e mi rincresceva, da qualche tempo, non leggere più nulla di tuo, non sentirti nemmeno al telefono. Eppure la tua fedeltà alla rivista era assoluta e sino alla fine abbiamo voluto che il tuo nome figurasse accanto a quello di Benedetta Papasogli in testa alla Rassegna bibliografica relativa al secondo Seicento. Era quella, col Settecento, la tua stagione preferita. La tua formazione filosofica ti invitava a chinarti con passione e severo spirito di ricerca sull'età d'oro del pensiero francese, da Descartes ai pensatori dei Lumi. Hai dato, su quel terreno, dei contributi fondamentali, ben prima del noto libro di Mauzi, i tuoi studi sulla nozione di bonheur hanno aperto una strada che molti, dopo, hanno inteso percorrere: vari anni or sono, un candidato alla libera docenza, dovendo svolgere il tema del malheur in non so quale epoca delle lettere francesi mi confessò di essersi preparato prendendo a modello i tuoi saggi e rovesciandone in qualche modo il contenuto: alludo a Moralistes del "bonheur" (1954), Virtù e critica della virtù nei moralisti francesi (1964), Montesquieu moralista (1965), Illuminismo, felicità, dolore (1969), Il serpente e la sirena (1972), Les tambours de Santerre (1986) e tanti altri, che sarebbe lungo elencare. Altro tuo campo di predilezione è stato quello di un genere tipicamente francese e tipico dell'età classica, da La Rochefoucauld a Vauvenargues, da Montesquieu a Chamfort: quello della "massima". Amavi le massime e i "moralisti" a tal punto che hai scritto anche un libro di riflessioni d'insieme sul genere (La maxime, 1968). Ma non eri un moralista nel senso predicatorio e parenetico, al contrario: la massima ti seduceva perché rovescia le credenze più facili e i luoghi comuni comodi e retorici, e lo fa con la stessa densa ed ellittica arguzia che a volte coglievo nel tuo ironico sorriso e nel lampo sagace dei tuoi occhi. Jean Dagen scriveva in una nota del 1989, dopo aver accennato alla tua «admirable culture européenne»: «C'est en moraliste que Corrado Rosso écrit sur les moralistes. On devine dans sa prose qui ne se refuse ni les images, ni un certain lyrisme, les signes contenus d'un engagement personnel». E c'è poi un terzo tuo terreno d'indagine, quello del concetto di uguaglianza, di cui ti sei occupato mirabilmente nel saggio Mythe de l'égalité et rayonnement des Lumières (1980): un tema che ti ha spinto a creare la collana Études sur l'égalité ed a sollecitare studi e ricerche in una direzione che non è, per altro, di natura sociopolitica, quanto di natura individuale e interiore: presentando il saggio di una tua ottima allieva e collaboratrice, Stella Gargantini, tu scrivevi in proposito: «Confiner l'égalité aux seules manifestations collectives et de groupes serait perdre de vue sa signification profonde et dynamique. Avant les groupes il existe des individus. L'égalité devient un fait collectif dans la mesure où elle fait battre les cœurs des hommes et des femmes qui font les groupes».

Anche tu lasci un gran vuoto nei nostri cuori. Torinese, amavi la nostra città, venivi volentieri a trovarci, partecipavi assiduamente alle nostre riunioni. Una volta, dalle finestre di casa mia, mi additasti con commozione la scuola elementare "Alfieri", dove da bambino hai studiato, come tutti i bambini del Cit Turin. Poi, sempre a Torino ti sei laureato in filosofia: scegliendo come tuo argomento, se ricordo bene, la "filosofia dei valori". I nomi di grandi maestri come Guzzo, Abbagnano, Pareyson ricorrevano sovente nella tua conversazione e forse sei sempre venuto volentieri a Torino perché il tuo era un ritorno alle origini, ai luoghi della tua infanzia e della tua formazione di studioso. Per l'ultima volta, forse, ci sei venuto nel 1994 proprio per rievocare la figura di Guzzo, ma forse mi sbaglio, sei certo venuto a trovarci anche più tardi, per qualcuna delle nostre riunioni redazionali. La tua vocazione di francesista 
è nata durante i soggiorni a Lione e a Rennes, come lettore d'italiano, poi a Marsiglia come direttore dell'Istituto di Cultura (prima eri stato a Stoccolma come addetto culturale). In quei luoghi, immagino, nacquero i tuoi progetti di ricerca, quelli che sfociarono nei libri a cui prima accennavo e anche in altri, relativi ad altre epoche, come i saggi e ritratti raccolti in Novecento francese ed europeo (1988).

Avevi creato una tua pubblicazione periodica, "Spicilegio moderno", ma ciò non ti impediva di dedicarti con passione ed impegno alla nostra Rivista, con cui hai collaborato sin dai suoi inizi facendo poi parte, dal '76, come tutti noi, del Comitato di Redazione. Anche di te, quindi, tutti noi sentiremo la penosa mancanza. Gli "Studi in onore" che il tuo dipartimento ti ha offerto anni or sono (La quête du bonheur et l'expression de la douleur dans la littérature et la pensée françaises. Mélanges offerts à Corrado Rosso, Genève, Droz, 1995) dicono bene quel che Tu hai significato per tutti i tuoi amici e colleghi, per i tuoi allievi, per la nostra Francesistica, per la cultura del nostro Paese. Ma ai tuoi meriti culturali si aggiungono, ed è difficile dirlo con parole adeguate, i tuoi meriti umani: la tua signorilità, il tuo tatto, la tua finezza e discrezione. Rimane, il tuo profilo di gentiluomo, di honnête homme, inciso per sempre nel nostro animo. 